\title{
Organization of the Mouse Motor Cortex Studied by Retrograde Tracing and Intracortical Microstimulation (ICMS) Mapping
}

\author{
Cheng-Xiang Li and Robert S. Waters
}

\begin{abstract}
The motor representation of the body musculature was studied in 11 adult mice by using ICMS. The motor responses elicited from both granular and agranular cortical fields showed that the mouse motor cortex is topographically organized; however, within the representation of individual body-parts the movements are multiply represented. In addition, several sites were encountered where more than one movement was elicited at the same stimulus threshold. The locations of pyramidal cells contributing axons to the pyramidal tract were examined by means of retrograde tracing with HRP injected into the cervical enlargement. This procedure labeled neurons only in lamina $\mathrm{V}$ in granular and agranular cortical fields. The similarities between the organization of motor cortex demonstrated in this study and the organization in the rat suggest that the rat and mouse share a common plan of rodent motor cortical organization.
\end{abstract}

\begin{abstract}
RÉSUMÉ: Étude de l'organisation du cortex moteur de la souris par traçage rétrograde et cartographie par microstimulation intracorticale (MSIC). La représentation motrice de la musculature corporelle a été étudiée chez 11 souris adultes au moyen de la MSIC. Les réponses motrices élicitées à partir des champs corticaux de cellules granuleuses et non-granuleuses ont montré que le cortex moteur de la souris a une organisation topographique; cependant, la représentation des mouvements est multiple au sein de la représentation de parties individuelles du corps. De plus, plusieurs sites ont été repérés où plus d'un mouvement étaient provoqués pour un même seuil de stimulation. La localisation des cellules pyramidales contribuant des axones au faisceau pyramidal a été examinée au moyen du traçage rétrograde en injectant du HRP dans le renflement cervical. Cette technique marquait seulement les neurones de la couche. $V$ dans les champs corticaux granuleux et agranuleux. Les similitudes entre l'organisation du cortex moteur démontrées par cette étude et l'organisation chez le rat suggèrent que le rat et la souris ont un plan commun d'organisation motrice corticale en tant que rongeurs.
\end{abstract}

Can. J. Neurol. Sci. 1991; 18: 28-38

The functional organization of the motor cortex in rat has been extensively studied ${ }^{1-6}$ by systematically sampling a large number of cortical sites using intracortical microstimulation (ICMS), a technique developed by Asanuma and colleagues.7-9 These maps of motor cortex describe a complete representation of movements for all body parts. Often the results of these studies were related to the cytoarchitecture of the region studied. 3,4,6 Although some ambiguities remain concerning the definition of primary motor cortex, the size and location of individual body part representations, and the extent of cytoarchitectonic boundaries, these studies have established some general parameters of motor organization of rat cortex.

In contrast, knowledge of the functional organization of mouse motor cortex lags considerably behind that of the rat even though the mouse somatosensory cortex and the barrel field in particular have been thoroughly studied using anatomical10-13 and physiological ${ }^{14-15}$ methods. In the present study, we examined the functional organization of mouse motor cortex using ICMS and related these findings to the underlying cytoarchitecture. We also studied the distribution of corticospinal neurons by using the retrograde tracer, horseradish peroxidase (HRP). Our results allow us to compare the motor cortical organization of the mouse with that of the rat and provide a motor map for a strain of mice $(\mathrm{C} 57 / \mathrm{BL}$ dy $2 \mathrm{j} / \mathrm{dy} 2 \mathrm{j})$ that will serve to lay the groundwork for subsequent investigations on the central consequences of motor disorders in genetic mutant strains of mice.

\section{METHODS}

Experiments were carried out on 38 adult mice (C57/BL dy $2 \mathrm{j} / \mathrm{dy} 2 \mathrm{j}$ ) weighing between $15-28 \mathrm{~g}$. Of this number, 6 mice were used to study cytoarchitecture, 5 animals were used in the

From the Department of Anatomy and Neurobiology, University of Tennessee, Memphis, College of Medicine, 875 Monroe Avenue, Memphis, TN, U.S.A. 38163

Received June 13, 1990. Accepted September 21, 1990

Reprint requests to: Robert S. Waters, Department of Anatomy and Neurobiology, University of Tennessee, Memphis, 875 Monroe Avenue, Memphis, Tennessee, U.S.A., 38163 
anatomical tracing experiment, and 27 were used for mapping motor responsive cortex.

Cytoarchitecture Mice $(\mathrm{n}=6)$ were anesthetized with nembutal $(40 \mathrm{mg} / \mathrm{kg}$, i.p.) and perfused with $0.9 \% \mathrm{NaCl}$ followed by $10 \%$ buffered formalin. Brains were removed and placed in a $20 \%$ sucrose/formalin solution until they were completely infiltrated with sucrose (approximately 2 days) as indicated by sinking to the bottom of the container. Next, the brains were frozen, coronal sections were cut at 60 -micron thickness, and the sections were stained with cresyl violet or thionin. Sections were reconstructed by using a camera lucida drawing system, and the cytoarchitectonic boundaries were superimposed onto each section. Section drawings were then aligned by fiducials, and each section drawing was entered into a computer by means of a digitizing tablet. A serial section reconstruction program (Eutectics) was used to generate a 3-dimensional reconstruction which could be rotated.

The cytoarchitecture of the rodent frontal cortex has been previously described. ${ }^{16-18}$ To permit a direct comparison between our results and previous studies on the functional organization of rat motor cortex, 3,6 we followed the organizational pattern described in rat and partitioned the frontal cortex into granular and agranular fields on the basis of presence or absence of granular layer IV, cell staining density in layer II, and width of layer V.

Corticospinal Tract Tracing Animals $(n=5)$ were anesthetized with an intraperitoneal injection of ketamine $(100 \mathrm{mg} / \mathrm{kg})$ and placed in a stereotaxic apparatus. Supplemental doses of ketamine ( $1 / 5$ th of the initial dose) were administered as needed to suppress hindlimb reflexes and spontaneous movements. Under aseptic conditions, the skin overlying the cervical spinal cord was opened and the underlying fascia and muscles were retracted. A laminectomy was performed and the second, third, or fourth cervical segment was exposed. The dura and spinal cord were hemisected with a knife fashioned from a scalpel blade. The area around the opening was packed in Gelfoam, and a $30 \%$ solution of HRP was injected into the transection through a glass pipette attached to a Hamilton syringe. A total of $5 \mu \mathrm{L}$ was injected into four separate locations along the mediolateral extent of the cut. At each site $1.25 \mu \mathrm{L}$ was injected over a period of $10 \mathrm{~min}$. Following the injection, the fascia and muscles were reattached and the skin was closed. Tissues around the surgical site were infiltrated with a long-lasting local anesthetic (Carbocaine).

After a survival period of $72 \mathrm{~h}$, mice were perfused with $0.9 \% \mathrm{NaCl}$, followed by a phosphate buffered solution containing $1.5 \%$ paraformaldehyde and $2 \%$ glutaraldehyde. The brain was placed in phosphate buffered saline (PBS) with $20 \%$ sucrose added, frozen, cut at 60-micron thickness, and incubated in diaminobenzidine (DAB) and $0.01 \%$ hydrogen peroxide solution. Sections were cover-slipped without counterstaining and examined under light and dark field optics. Each section was reconstructed by using a camera lucida drawing system, and the locations of labeled cells were plotted on the reconstructed drawing. Three dimensional reconstructions were generated as described above for the cytoarchitectural analysis. Following reconstruction, coverslips were removed and sections were counter-stained with cresyl violet.

Motor Mapping All results of the ICMS mapping experiments reported below were obtained from animals for which a complete body map was generated from the same individual. The animals $(n=27)$ were anesthetized initially with ketamine $(100 \mathrm{mg} / \mathrm{kg}$, i.p.). This was supplemented with $1 / \mathrm{sth}$ of the initial dose as needed to suppress hindlimb reflexes and spontaneous movement. The hair on the contralateral side of the body was shaved to facilitate the examination of movements. Animals were placed in a modified stereotaxic frame to stabilize the head, and the body was elevated on a platform so that the limbs were suspended. A mirror was positioned in front of the platform to permit observation of the underside of the head, neck, and limbs. A water-circulating heating pad maintained the animal's body temperature between $37-39^{\circ} \mathrm{C}$. The cisterna magna was opened and allowed to drain to reduce pulsations of the brain. An incision was made in the skin over the right parietal cortex, the underlying bone removed, and the dura exposed. A well, formed around the craniotomy with dental impression wax, was filled with a heavy-grade silicon fluid $(10,000 \mathrm{cST})$ to prevent drying and further reduce pulsations. A photograph of the cortical surface was taken through the dura and was used to record the location of electrode penetrations. Since the dura was thin enough to view the cortical surface, no electrode penetrations were made in the vicinity of large surface vessels.

A tungsten-in-glass microelectrode attached to a microdrive (Narishige) was employed to deliver a train of cathodal stimulating pulses ( 12 pulses/train, $0.2 \mathrm{~ms}$ duration, $300 \mathrm{~Hz}$ ) to elicit motor responses. The amplitude of stimulation current (constant voltage source) was monitored on an oscilloscope and measured as the voltage drop across a $10-\mathrm{K} \Omega$ resistor in series with the return lead to the stimulator. The initial search stimulus was set at $30 \mu \mathrm{A}$. Threshold current was defined as the lowest current that produced a consistent movement. Threshold was determined by first eliciting a movement, reducing the current until the movement ceased, increasing the current until the movement reappeared, and reproducing the response on at least three consecutive stimulus presentations. However, if a motor response was not evoked at $30 \mu \mathrm{A}$, the current was increased in steps of $10 \mu \mathrm{A}$ until a response occurred or a maximum level of $50 \mu \mathrm{A}$ was reached.

In a series of preliminary experiments, ICMS was delivered throughout the entire depth of an electrode penetration to determine the depth at which thresholds were minimal. In all subsequent experiments, ICMS was delivered at an initial depth of 0.7 $\mathrm{mm}$, since stimulation at this depth generally yielded the lowest thresholds. However, if no motor movement was observed at this depth, the electrode was advanced to a depth of $0.8 \mathrm{~mm}$, and the stimulation sequence was repeated. A depth of 0.7-0.8 $\mathrm{mm}$ corresponds to deep layer $\mathrm{V}$ and superficial layer VI. Motor mapping along the midline was often carried to a depth of 1.0 $\mathrm{mm}$. Each electrode penetration was separated from the others by 250 microns; however, in those cases where the electrode encroached upon surface blood vessels, the penetrations could be separated by $400-500$ microns. All electrode penetrations were made perpendicular to the surface of the cortex. Bregma was used as the zero reference point, and all electrode penetrations were made in relationship to that reference. The edge of the motor map was defined as those locations where two or more successive unresponsive stimulating sites were encountered.

Electrolytic lesions ( $10 \mu \mathrm{A}$ for $10 \mathrm{~s})$ were made at the end of selected electrode tracks and/or at the end of the experiment to mark the location of stimulation sites for later histological reconstruction. Mice were given an overdose of sodium pentobarbital $(50 \mathrm{mg} / \mathrm{kg})$ and perfused through the left ventricle with a $0.9 \%$ solution of $\mathrm{NaCl}$ followed by $10 \%$ buffered formalin. Brains were removed and placed in a $20 \%$ solution of sucrose in 
formalin. After infiltration of the tissue, frozen sections were cut at 60-micron thickness, mounted on gelatin coated slides, and stained with cresyl violet. The electrode penetrations and lesions were reconstructed with reference to cytoarchitecturt. For each cortical stimulation site, an entry was recorded on the data sheet that included the observed movement and the threshold stimulus value. Stimulation sites in which no movements were observed were also recorded. The data were then used to generate a map of the motor representation and the movement(s) elicited at each site.

An idealized summary map was generated by superimposing the outline of areas representing body-parts on each other and drawing a line that in our estimation best represented the common features of the organization. Although we did not explore the details of the motor representation of the face and tongue, the general location of these body-part representations has been noted on the map, even though this addition does not reflect the full extent of the representation.

\section{RESULTS}

Cytoarchitecture Figure 1 shows a photomicrograph of two coronal sections from the same animal, illustrating the cytoarchitectonic characteristics of the granular and agranular fields. The photomicrograph in Figure $1 \mathrm{~A}$ was taken from a section approximately $1.2 \mathrm{~mm}$ anterior to bregma. Note the presence of a densely stained layer II located along the midline that continues onto the gyrus, and the less differentiated deeper layers lying along the midline. These two traits characterize agranular medial field $(\mathrm{AgM}))^{3.6}$ Layer $\mathrm{V}$ is quite clear in the more lateral part of $\mathrm{AgM}$. In the adjacent agranular lateral field (AgL), layer II does not stain as darkly as it does in AgM. Layer IV, if present, consists of only a few cells, and layer $V$ is quite wide. On the other hand, granule cells can be clearly seen in the neighboring granular primary somatosensory cortex, GSI, where they lie above the less cell-dense layer $\mathrm{V}$. The photomicrograph in Figure $1 \mathrm{~B}$ is a section taken from cortex approximately $1.2 \mathrm{~mm}$ posterior to bregma. This section contains a clearly demarcated $\mathrm{AgM}$ field that is characterized by concentrated staining in layer II immediately adjacent to the midline. Note the presence of a densely packed layer IV in the GSI which appears uniform along its extent. The appearance of tightly packed granule cells in layer IV may be contrasted with the less cell-dense layer V.

While individual differences in the dimensions of each cytoarchitectonic field were observed among animals, nonetheless a general overall pattern emerged. Figure 2 illustrates a computergenerated reconstruction of the locations of the granular and agranular regions for one animal. This figure was produced by digitizing the outline and location of granular and agranular fields in 60 micron-thick sections and aligning the sections to produce a serial reconstruction. AgM is located in the anteriormost part of cortex, where it extends across the entire mediallateral surface and continues along the midline, tapering off at a point posterior to bregma. AgL is located lateral to $\mathrm{AgM}$ and is sandwiched between this field and the more laterally located GSI. Rostrally, AgL is quite wide but tapers toward the midline as it approaches bregma. GSI lies most lateral and extends over the lateral convexity and down the lateral side of the cortex.

Corticospinal Tract Tracing To understand the relationship between these cytoarchitectonic fields and the corticospinal projecting neurons, the distribution of corticospinal labeled neurons in the cortex was plotted in a similar manner for 5 animals. Two cases are shown in Figure 3. In the case illustrated in Figure 3A,

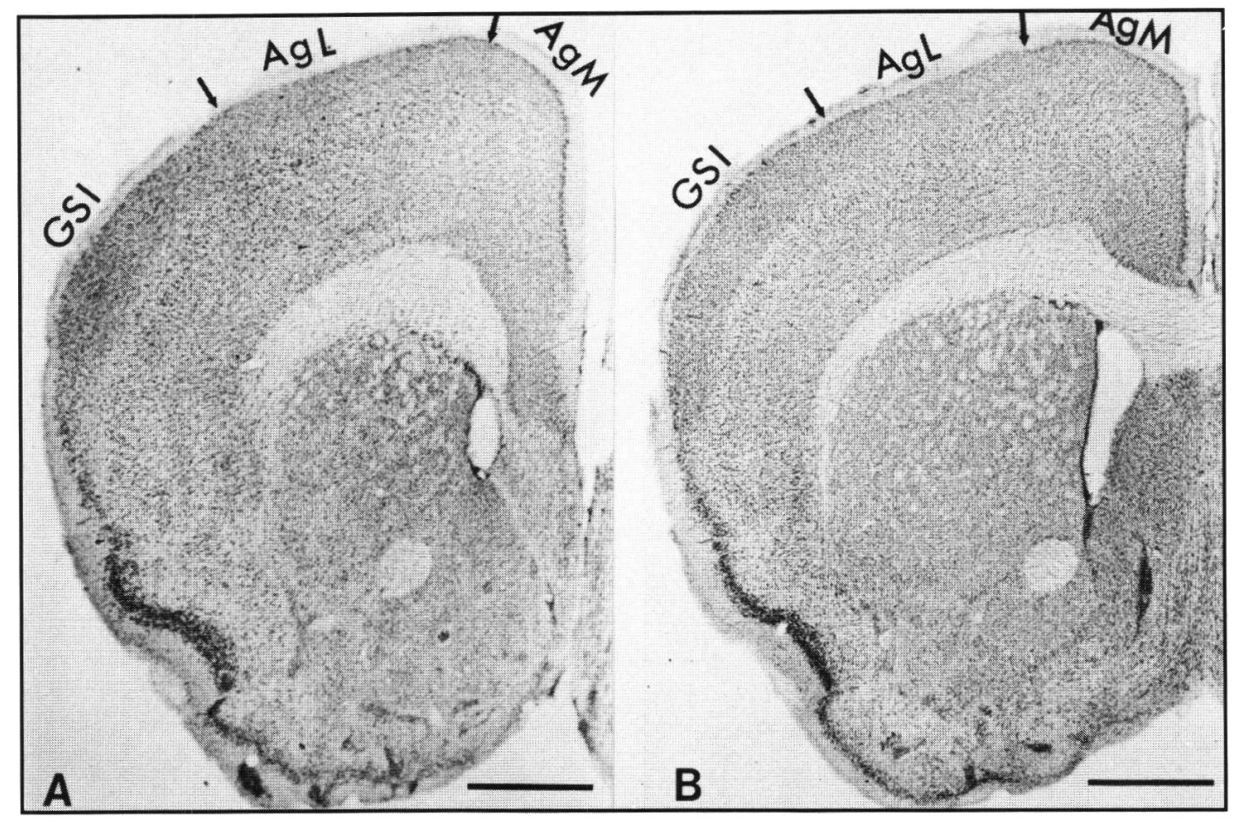

Figure I A,B - Photomicrographs of coronal sections taken from the same animal illustrating the cytoarchitecture of mouse cortex. A Section taken from approximately $1.2 \mathrm{~mm}$ anterior to bregma. Agranular field is subdivided into medial ( $\mathrm{AgM}$ ) and lateral $(\mathrm{AgL})$ divisions. The granular field is designated GSI to indicate that it is also part of the primary somatosensory cortex (SI). B Section taken from approximately $1.2 \mathrm{~mm}$ posterior to bregma illustrating the granular and agranular fields. Scale bar $=1 \mathrm{~mm}$. 


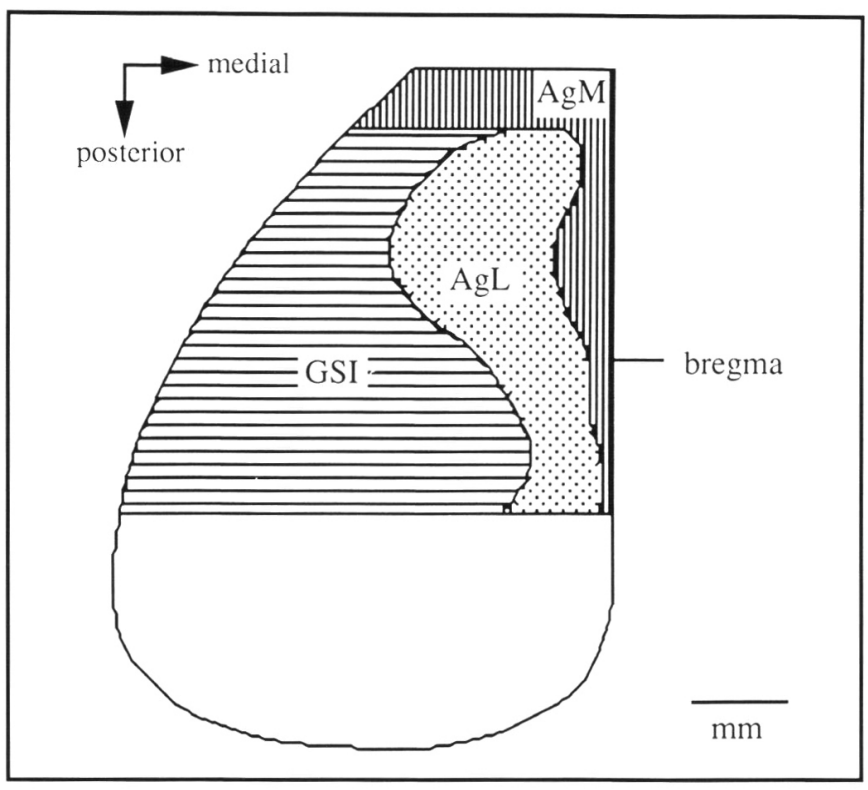

Figure $2-$ Computer-generated reconstruction of 60-micron thick coronal sections showing the relationship between the location of granular and agranular cortical fields. AgM is located in the dorsomedial part of the cortex and extends caudally along the midline. $\mathrm{AgL}$ is bordered on the rostromedial side by $\mathrm{AgM}$ and on the lateral side by GSI. Scale bar $=1 \mathrm{~mm}$.
HRP was injected into the second cervical segment (C-2), while in Figure $3 \mathrm{~B}$ the injection was made into the fourth cervical segment $(\mathrm{C}-4)$. In no case did the injection site extend rostrally beyond $\mathrm{C}-2$. This figure illustrates that HRP-labeled neurons were restricted to lamina $\mathrm{V}$ in both the granular and the agranular fields. The location of the representation of labeled neurons within each cortical field is shown in Figure 3; however, the largest number of labeled cells was found in AgL and GSI. Arrows are inserted in the figure at the boundaries of each cortical field. In the rostral-most sections, labeled neurons were found in $\mathrm{AgL}$ and within $\mathrm{AgM}$. Labeled neurons in AgM were, in large part, found beneath the gyral convexity and gyral crown, but no labeled cells were found in cortex adjacent to the midline. More caudal sections were characterized by labeling in $\mathrm{AgL}$ and GSI; however, only a few labeled neurons were found in AgM. Labeled neurons were always pyramidal-shaped (not shown) but differed in size.

A computer-microscope plotting system (Eutectics) was used to generate a 3-dimensional reconstruction that enabled the data to be rotated so that the pattern of labeled cells could be viewed directly from the front and from above. The distribution of labeled cells for the two cases is shown in Figures 4A and 4B. The upper parts of each figure are drawn from a horizontal perspective, as if the observer were standing directly anterior to the cortex and looking in a caudal direction at a series of coronal sections. From this perspective, it is clear that labeled cells were

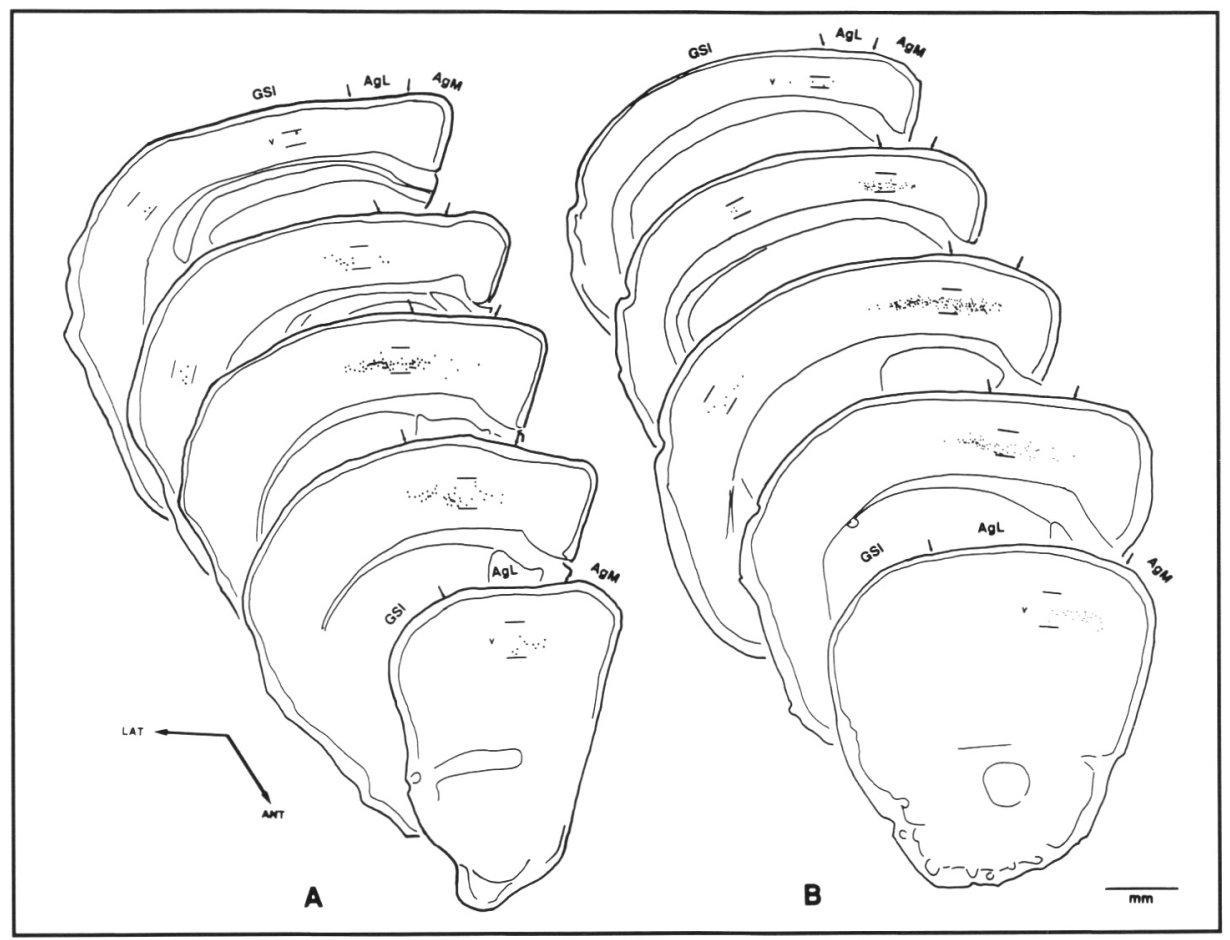

Figure $3 A, B$ - Five reconstructed line drawings of photomicrographs from two animals showing the location of HRP labeled cells in mouse cortex. The locations of the granular and agranular fields are indicated by arrows at the top of each figure. A second region of labeling was found in the lateral-most part of the cortex. All labeled cells were localized in lamina $V$. Lamina $V$ boundaries are indicated by parallel lines bracketing the labeling. Scale bar $=1 \mathrm{~mm}$. 
found deep within the cortex; all labeled cells were confirmed to lie entirely within lamina V. Labeled neurons were found in 3 distinct regions of cortex. This can be readily seen in the dorsal view shown in the lower part of Figure 4A and 4B. One region lies in the rostral part of the dorsomedial cortex (letter " $C$ "), the second region lies caudal in the dorsomedial cortex (letter " $A$ "), and the third region lies laterally in parietal cortex (letter "B"). Figure 4 (upper) shows the locations of these regions. The lower part of Figure 4 plots the same data, but this time the plot is rotated as if the viewer is looking down at the overall pattern. The arrow along the midline indicates the location of bregma. From this perspective, it appears that labeled neurons are localized into medial and lateral regions. The medial region is located near the midline and can be separated into rostral and caudal regions. The caudal region, indicated by " $A$ ", contains the densest labeling and is separated from a lighter labeled rostral region, indicated by "C", by a gap containing few or no neurons. In the first example illustrated in Figure 4A, labeled cells in region " $A$ " can be seen to extend without interruption into region " $C$ "; however, in the case illustrated in Figure 4B, a clear gap can be seen between the two components. The third region of cells, indicated by "B", lies lateral in parietal cortex and is separated by a gap between the dorsomedial regions.

Intracortical Microstimulation A total of 1361 electrode penetrations were made in 11 adult mice to explore the details of the motor representation of the vibrissae, forelimb-shoulder, and hindlimb-trunk. The representation of the face (lips, jaw) and tongue movements was not studied in detail.

The general organization of the map for movements of the vibrissae, forelimb, and hindlimb is shown for three animals in
Figures $5,6,7$. These were chosen to emphasize the variability observed among animals. Each map was generated by using the lowest intensity ICMS that evoked a movement. Those stimulating sites with thresholds between $<1$ and $25 \mu \mathrm{A}$ are indicated with filled circles or uppercase letters, and stimulating sites with thresholds between 26 and $50 \mu \mathrm{A}$ are designated with open circles or lowercase letters. The relationship between cytoarchitecture and stimulation sites is shown for two animals; cytoarchitectonic boundaries were less clear in the third animal and therefore were not included in the figure.

Vibrissae Representation Figure 5 illustrates the location of the sites producing movements of the vibrissae for the three cases. The representation extends approximately $3.5 \mathrm{~mm}$ along the midline, beginning $2.0-2.5 \mathrm{~mm}$ anterior to bregma and continuing to a location $0.5-1.5 \mathrm{~mm}$ posterior to bregma. The tip of the rostral pole of frontal cortex lies approximately $3.0 \mathrm{~mm}$ anterior to bregma, and because of difficulty in accessing this region, we did not explore the anterior-most $0.2 \mathrm{~mm}$ in each animal. The vibrissae representation consists of at least 2 and possibly 3 separate maps. Each map (indicated by dashed lines) is separated by cortex where vibrissae movements were not elicited with stimulating currents less than or equal to $50 \mu \mathrm{A}$. One map is located in the rostral-most part of $\mathrm{AgM}$ and $\mathrm{AgL}$ and consists of a restricted region of less than $1 \mathrm{~mm}^{2}$. This representation is separated from a caudal map that is found largely in $\mathrm{AgL}$ and borders on AgM along its midline extent. In the case illustrated in Figure 5A, the caudal map was discontinuous and a third representation was found in which vibrissae movements were evoked at a location $1.5 \mathrm{~mm}$ posterior to bregma.

Medial to the vibrissae representation, the cortex was unresponsive to ICMS; however eyelid movements can be elicited

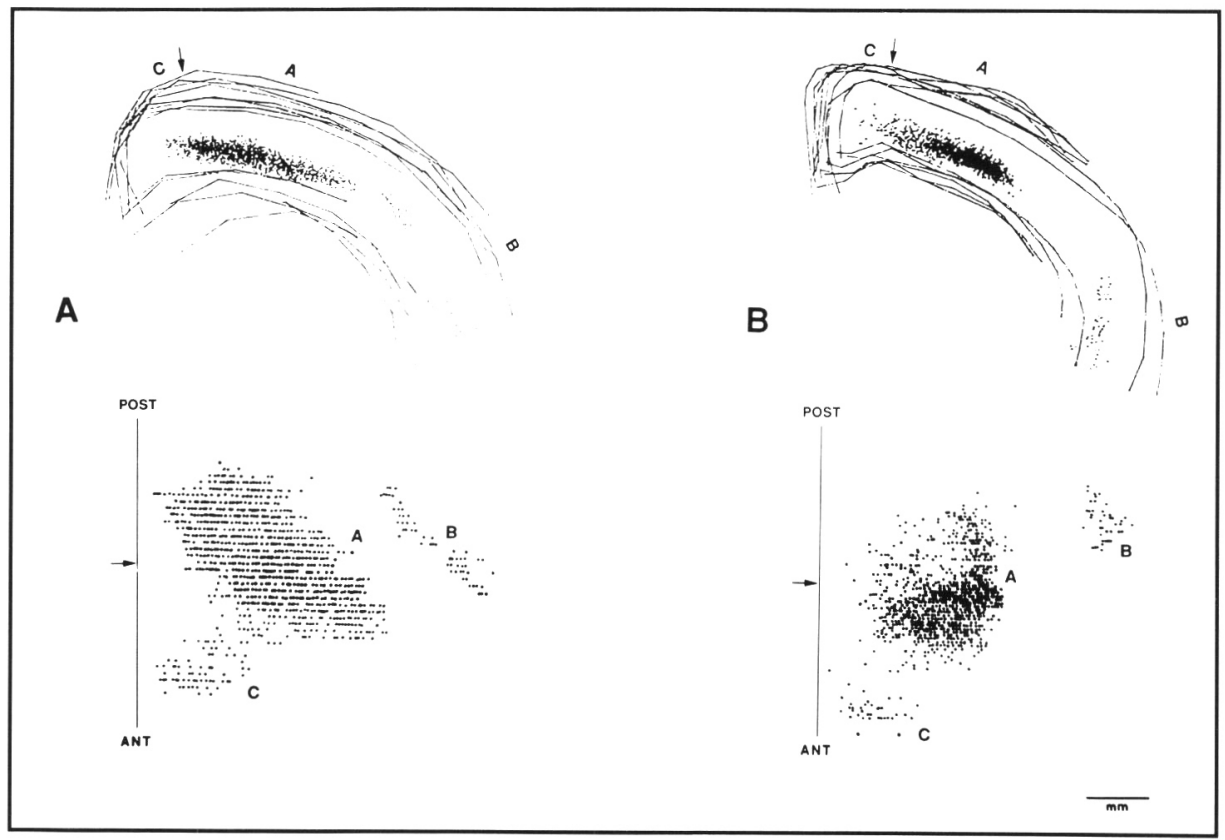

Figure $4 A, B$ - Computer generated reconstruction of HRP labeled neurons for the two cases illustrated in Figure 3. Upper parts of the figure are viewed from a perspective as if the observer were standing anterior to the cortex and looking in a posterior direction at the arrangement of aligned coronal sections. Arrows indicate the approximate boundary between rostral (" $C$ ") and caudal (" $A$ ") bands. The labeling in lateral cortex is indicated by " $B$ ". Lower part of the figure is to be viewed as if the observer were looking down upon the cortex at the overall distribution of labeling for the two animals. Arrows along the midline indicate the location of bregma. Lettering is the same as in the upper part of figure. Scale $=1 \mathrm{~mm}$. 
from this region in rat. 1,6,19,20 The vibrissae representation on the rostrolateral side is bordered by the face and tongue representations. On the lateral side, the vibrissae representation borders and/or overlaps the forelimb-shoulder and hindlimb-trunk representations. We will describe this overlap in a later section.

Stimulating intensities as low as $5 \mu \mathrm{A}$ were sufficient to elicit vibrissae deflection from both ipsilateral and contralateral whiskers. Vibrissae movements were observed from small clusters (3-5 whiskers) as well as deflection of the entire mystacial pad. Ipsilateral, contralateral, and bilateral movements were seen in all animals; however, bilateral movements were most common. In those cases where bilateral movements were present, we often observed more vigorous contralateral movements. Vibrissae responses were elicited from cortical sites in AgM and $\mathrm{AgL}$; however, vibrissae movements were most often activated from the region bordering $\mathrm{AgM}$ and $\mathrm{AgL}$ (Figures $5 \mathrm{~B}$ and $5 \mathrm{C}$ ).

Forelimb Representation Individual motor maps illustrating the location of the forelimb representation are shown in Figure 6 . It may be seen from these maps that the forelimb representation is located, in large part, in the region of cortex around bregma and immediately anterior (1.0-1.5 mm) to bregma. A close inspection of the data reveals that digit or elbow movements could also be elicited at sites anterior to this representation, suggesting that the forelimb may have two separate maps.

In order to determine whether an internal organization exists within the larger forelimb map, we plotted the location of the motor representation of the digits, wrist, and elbow. Individual differences were seen between the forelimb maps (Figure 6), but we did not observe a clear pattern of internal organization within any one map; rather, the same movement was represented in more than one site. These locations were often separated from one another by distances of several hundred microns. Forelimb movements were elicited from both AgL and GSI cortex; however, they were most often encountered in AgL. Nonetheless, when movements were observed in GSI, they were elicited with threshold values similar to those eliciting the same movement in AgL. Although not illustrated in the figure, we observed an equal number of flexor and extensor movements for wrist and elbow; however, digit movements were restricted to extension of two or more digits. Single digit movement was not observed.

Hindlimb Representation Figure 7 shows a reconstruction of sites where hindlimb movements were activated with stimulating currents of $50 \mu \mathrm{A}$ or less. These movements were generally elicited from locations posterior to bregma and often extended $2.5 \mathrm{~mm}$ in the same direction. In two cases, illustrated in Figures $7 \mathrm{~B}$ and $7 \mathrm{C}$, hindlimb movements could be elicited anterior to bregma. These movements were generally restricied to the hip and were found in only a few penetrations in an area of less than $1 \mathrm{~mm}^{2}$.

To determine whether an internal organization existed for individual movements within the hindlimb representation, we plotted the locations of the hip, knee, and toe. No obvious individual pattern of organization could be seen; rather, the same
A

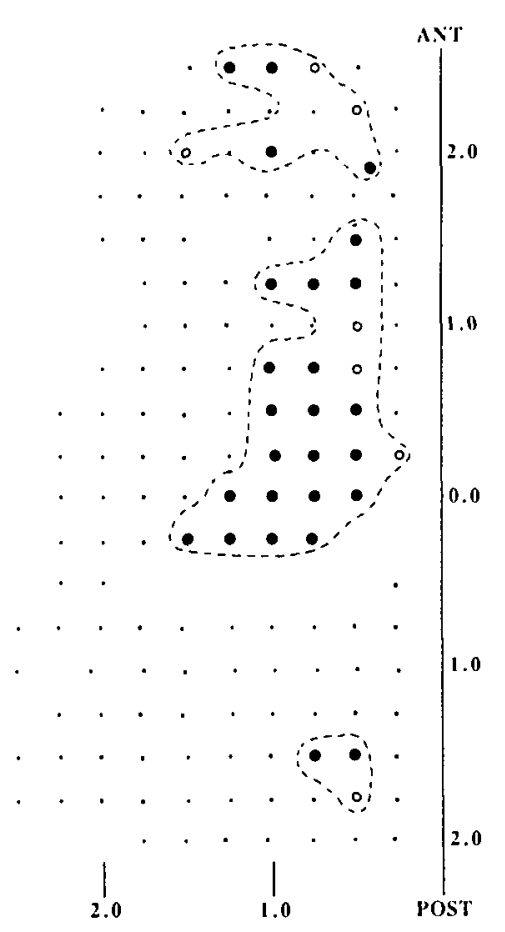

B

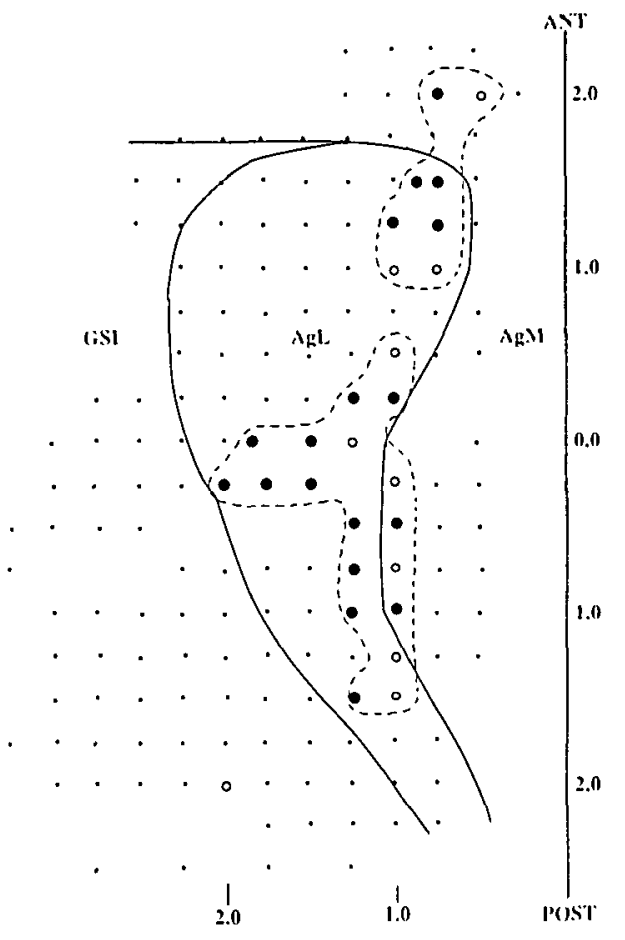

C

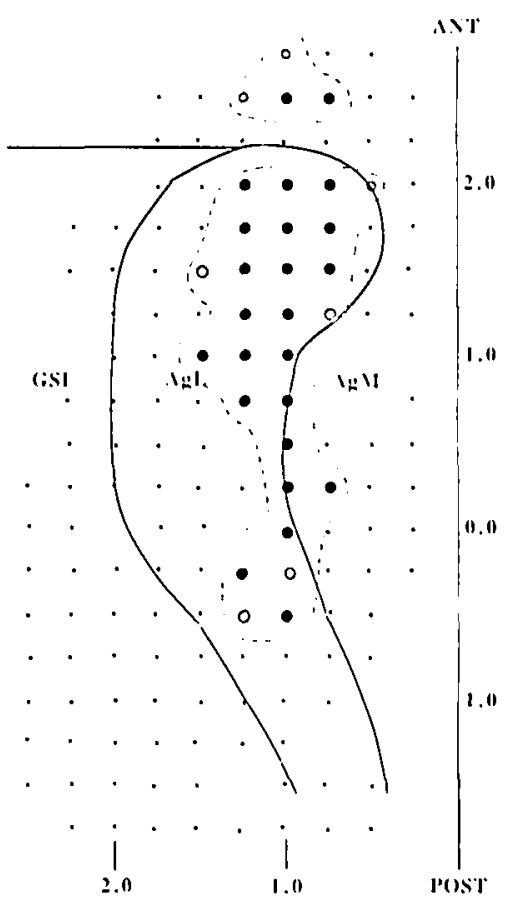

Figure 5 A-C - Line drawing reconstruction of the distribution of sites in the motor responsive cortex where vibrissae movements were generated with ICMS at threshold values of $50 \mu A$ or less. Three representative cases are shown in these figures. Filled circles indicate threshold values below $26 \mu \mathrm{A}$ while open circles indicate thresholds between 26-50 $\mu \mathrm{A}$. Penetration sites where the vibrissae were not activated at threshold values at or below $50 \mu \mathrm{A}$ are indicated by dots. Dashed lines show separate vibrissa maps. Empty locations within the matrix of dots indicate locations where blood vessels were found. The cortex was sampled at 250 micron intervals. Figures 5 B.C show the relationship benveen the stimularing sites and the cytoarchitectonic fields. $A g M=$ agranular medial; $A g L=$ agranular lateral; $G S I$ = granular somatosensory cortex. 
movement could be elicited at many different locations within the hindlimb representation. Flexion and extension movements were observed for both toe and knee, although movements were largely restricted to flexion-type movements. Toe movements were observed to act only in unison with one or more other toes.

Overall Representation The overall motor organization of the mouse cortex is summarized in Figure 8 as a composite of the three previously illustrated cases. Data points were included for stimulating threshold values of less than or equal to $50 \mu \mathrm{A}$. Not all movements were elicited from each animal, and individual differences in the representation of movements were apparent among maps. For example, neck movements were elicited in only one of the three cases at threshold (see Figure 8C); however, these movements were observed in the other two animals at suprathreshold values. Nose twitch was observed in only one of the three cases (Figure $8 \mathrm{C}$ ), and this response was seen only in the most rostral part of the representation. Movements of the neck, trunk, and tail were also elicited, but it was not uncommon for these responses to be accompanied by one or more other movements. Face movements were encountered only in the most anterolateral portion of the representation, and their appearance generally signalled the lateral border of the vibrissae and/or forelimb representations. Tongue movements were encountered within the face representation, but these responses were found mainly at more lateral locations. Neither the face nor tongue representation was studied in detail, however.
The topographical organization of body parts and the geaeral orientation of the map running along the anterior-posterior axis can be readily seen from these figures. The major components are the vibrissae, limbs, and face/tongue representations. In Figures 5-7, the individual representations of the vibrissae and limbs are shown, but when representations of other body parts are included in the map (Figure 8 ) a further degree of complexity may be seen as some degree of overlap. Two types of data points were encountered. The first and most common were sites where a single movement was elicited at threshold. The second type was unexpected: many sites were encountered where more than one movement was elicited at the same threshold. This overlap of responses was observed in every animal studied, leading to the conclusion that it was a general feature of the organization of the mouse motor cortex. Inspection of Figure 8 reveals that overlap was found between each major body part with the exception of the face representation, which did not overlap with either limbs or vibrissae. Overlap of forelimb and hindlimb representations generally occurred at their common border (Figures $8 \mathrm{~A}$ and $8 \mathrm{~B}$ ). However, overlap at borders can be compared to cases (see Figure 8C) where overlap occurred elsewhere. For example, the vibrissae were moved by stimuli between limbs and axial representation, but overlap was most likely to occur along the region bordering the two representations.

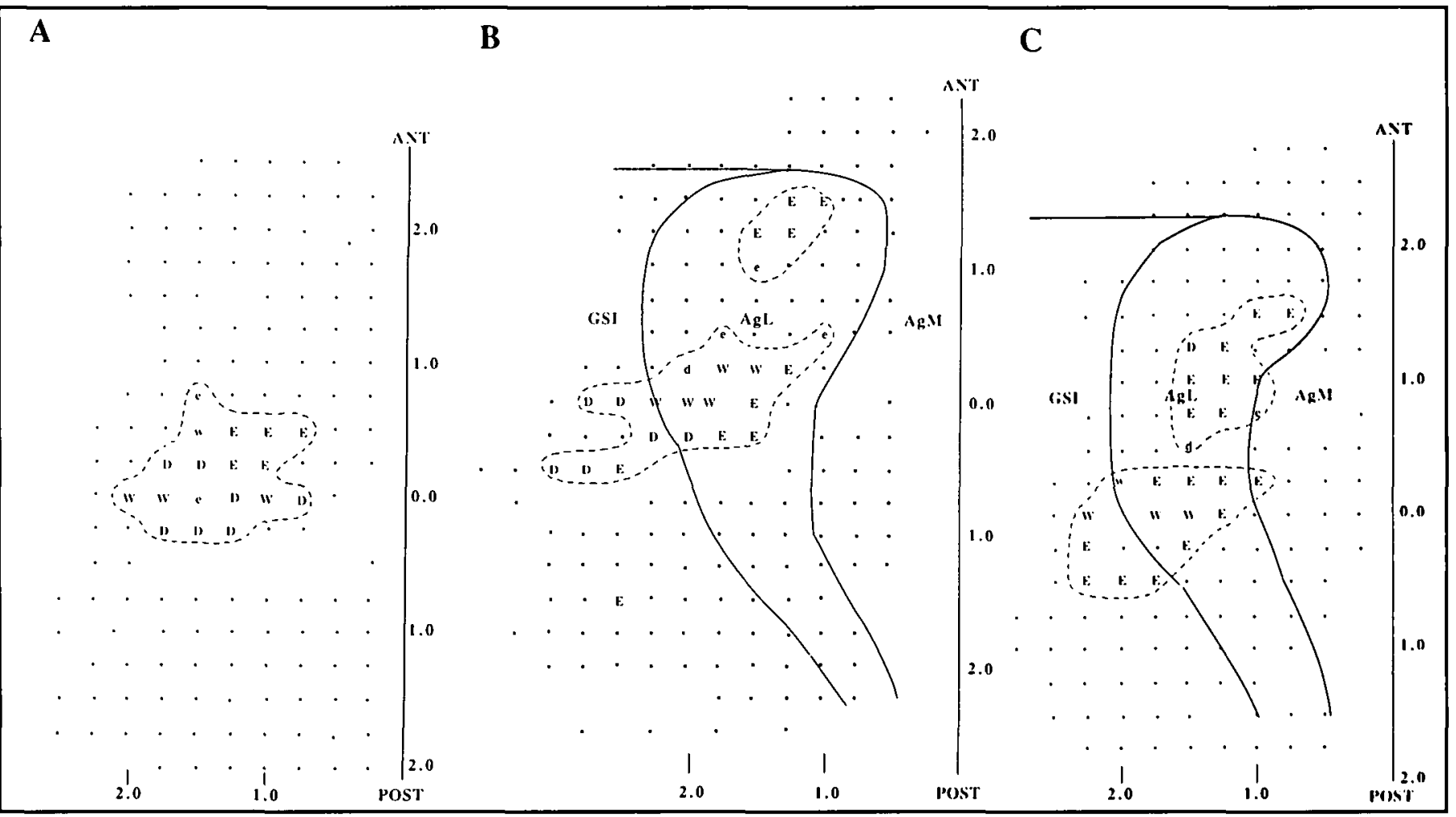

Figure 6 A-C - Line drawing reconstruction of the distribution of sites in motor cortex where forelimb movements were generated with thresholds at or below $50 \mu \mathrm{A}$ for the three representative cases shown in Figure 6. Recording sites where forelimb movements were activated below $26 \mu A$ are indicated by uppercase letters; threshold values between 26-50 $\mu \mathrm{A}$ are indicated by lowercase letters. Dashed lines indicate separate forelimb representations. $D(d)=$ digit $;(w)=$ wrist $; E(e)=$ elbow: 


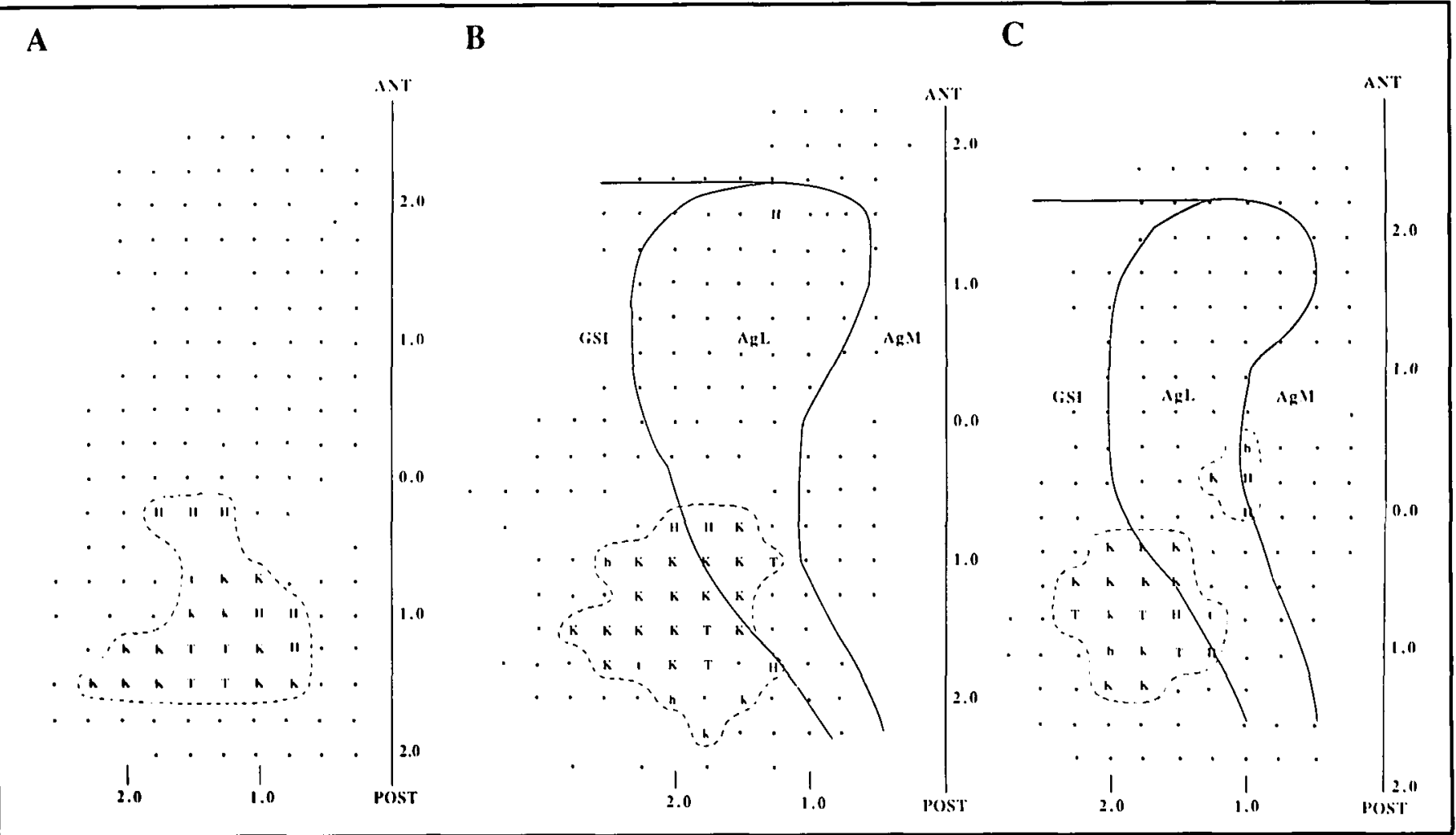

Figure 7 A-C - Distribution of motor effective sites for the representation of the hindlimb for the three representative cases shown in Figures 5 and 6. Uppercase letters indicate thresholds below $26 \mu \mathrm{A}$ while lowercase letters indicate thresholds between 26-50 $\mu \mathrm{A}$. Dashed lines indicate hindlimb representations that are separated by sites where ICMS was ineffective in evoking hindlimb movements. $T(t)=t o e ; K(k)=k n e e ; H(h)=h i p$.

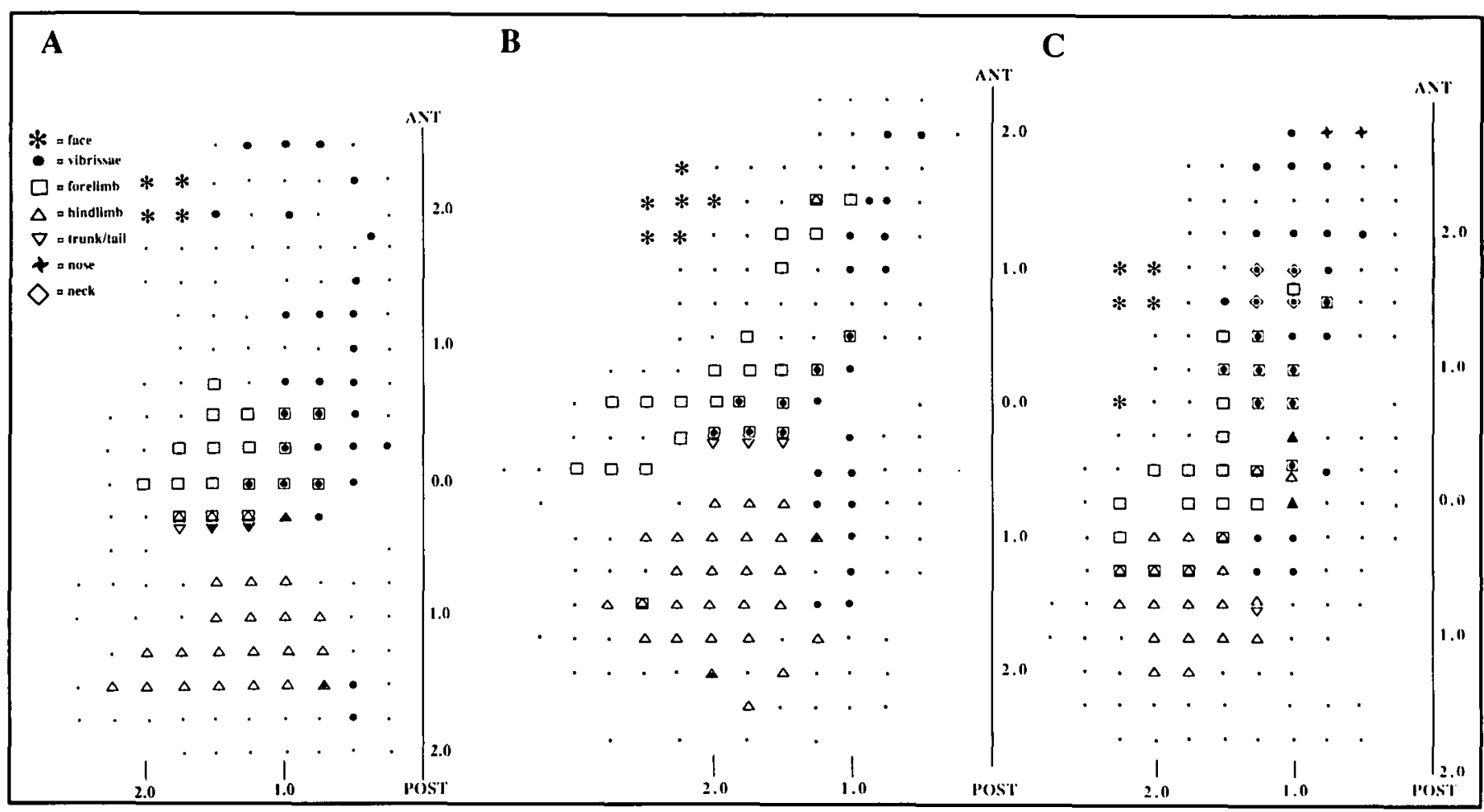

Figure 8 A-C - Overall distribution of movements for the three cases illustrated in Figures 5-8. Note the fact that several sites were acrivated where more than one muscle-movement was evoked. All responses were elicited with threshold stimulation values of $50 \mu A$ or less. 


\section{Discussion}

The present study is the first to examine the motor cortical organization in normal mouse by using retrograde tracing and ICMS techniques. The results show that both corticospinal tract neurons and the motor map are located in agranular and granular cortical fields.

Cytoarchitecture The cytoarchitecture of the mouse cortex has been described in detail by a number of investigators. ${ }^{16-18}$ However, since one goal of the present study was to examine the motor organization in mouse and compare our findings to those in rat, we characterized the mouse cortex in more general terms of agranular and granular fields as previously described in rat. 3.6 Our results, however, are consistent with the detailed architectonic maps of mouse cortex described by Caviness, 18 whereby the portion of $\mathrm{AgM}$ that lies along the midline corresponds to the dorsomedial field of area 8; the rostral-most part of AgM probably corresponds to parts of areas 6 and 10. Area 6 is described as a motor area that consists of a high concentration of cells in layer II and a wide layer $\mathrm{V}$ that contains large pyramidal cells and is similar to AgL. GSI, which consists of the primary somatosensory cortex, is similar to areas 1 and 3 .

The classification of motor responsive cortex into granular and agranular fields is based primarily on the presence or absence of granular cells in layer IV, degree of staining in layer II, and the breath of layer $\mathrm{V}$. In mouse as well as in rat cortex, AgM lies medial on the dorsal surface and extends down the medial wall toward the midline. Anteriorly, it occupies the dorsal part of the rostral pole and tapers sharply toward the midline as it approaches bregma. In both species, AgM is characterized by a darkly staining layer II and a lighter, uniformly staining layer III, and AgL contains a relatively lighter layer II and a wide layer V. The granular field, GSI, is identified by the presence of a welldefined granular layer IV, which is easily distinguished from the lighter staining layer V (see Figure 1). The major difference in our observations from those reported in rat is that we were unable to discern regions of lighter labeling in our sections within the granular field, which a number of investigators have described as a dysgranular zone..$^{3.4,21}$ In the rat, for example, Chapin and Lin $^{21}$ illustrate the dysgranular zone in flattened-tangential sections and describe it as that area lying between the face and forelimb representation that is unresponsive to somatic stimulation. Since our tissue was sectioned in the coronal plane and was not flattened, this zone may not be as clearly defined in our sections.

Corticospinal Tract The results suggest that HRP injected into the cervical segment labels corticospinal tract neurons into three separate cortical regions. Two of these regions are located in dorsomedial cortex and are separated by a gap containing few or no cells. We also identified a third region of labeling in the lateral part of granular cortex. There is no continuity of labeling between these two regions.

Few investigators have used retrograde tracing methods to study the corticospinal tract in the mouse. Those investigators who have used this method have focused, in large part, on comparing labeled cell types in normal and mutant mice. ${ }^{22}$ To the best of our knowledge, the present study is the first to describe the overall pattern of labeling of corticospinal tract neurons in the mouse. In contrast, the corticospinal tract has been well studied in the rat. $3,23-26$ Hicks and D'Amato ${ }^{25}$ first injected HRP into locations in the cervical and lumbar segments and reported labeled cells in a rostral and caudal band in a dorsomedial region of frontal cortex. These two bands were separated from each other by a gap where no labeled cells were found. They did not report labeling in other regions of the cortex. In a more recent study in rat, Donoghue and Wise ${ }^{3}$ reported labeled cells in the dorsomedial (AgL and AgM), second somatosensory cortex (SII), and posterior parietal cortex following injection of HRP into the cervical enlargement. Our results differed from those of Donoghue and $\mathrm{Wise}^{3}$ in that we found very few labeled cells in $\mathrm{AgM}$. This difference in findings may reflect the fact that we used a less sensitive chromogen in processing our tissue. In perhaps the most ambitious study to date, Nudo and Masterton27 studied the pattern of corticospinal organization in 23 different groups of mammals following deposit of HRP flakes into cut fibers in the cervical segment. Although they did not examine the mouse, they did study a closely related species, the pine vole. They described three zones of labeling (designated "A", "B", " $C$ ") in the pine vole, two of which were localized in the dorsomedial cortex (zones " $A$ " and " $C$ ") and the third in the lateral cortex (zone "B"). This pattern of labeling was similar to the pattern observed in our study, and therefore we characterized the labeling observed in the present study in terms of the zones described by Nudo and Masterton. 27

The one question that remains to be determined is the nature of the representation in the rostral part of dorsomedial cortex. Since this region consists of both $\mathrm{AgM}$ and $\mathrm{AgL}$ fields, we are tempted to speculate that movements of the neck and upperbody may be represented in this region. It is also possible that the rostral region corresponds to the supplementary motor cortex, and possibly higher levels of stimulation than those used in the present study will be needed to explore this region.

Intracortical Microstimulation We used the microstimulation technique developed by Asanuma and colleagues ${ }^{7.9}$ to map the motor cortex. Although this technique is not without controversy, $28-32$ it has nonetheless provided important insight into our understanding of the organization of the motor cortex.

A representative summary of the motor organization of the mouse cortex showing the typical features of the overall organization of motor responsive cortex is shown in Figure 9. From this idealized summary it is evident that the map, excluding the tongue and face representations, extends approximately $4.5 \mathrm{~mm}$ in an anteroposterior direction and approximately $2.5 \mathrm{~mm}$ in the mediolateral direction. This corresponds to the general orientation reported by each group of investigators who used microstimulation to map the rat motor cortex.1,3-6 Within our overall map, the representations of the vibrissae, forelimb, hindlimb, and face/tongue consumed the largest cortical space. These same bodypart representations were also the largest part of the rat motor map.1,3-6 The complete complement of movements was not observed at threshold levels of stimulation in every animal examined; for example, the nose was not represented in all animals, and in others cases where it was found, it was not the lowest threshold response at a particular site.

The mouse motor cortex is topographically organized. We observed multiple representations of individual body parts for each animal studied. In some cases we observed three separate representations of the vibrissae, whereas a dual representation was found for both the forelimb and the hindlimb (see Figure 9). The anterior-most representation comprised a smaller amount of cortex often measuring less than $1 \mathrm{~mm}^{2}$. A similar finding of 
multiple representation of individual body parts has also been reported in rat. $4-6,33$

Unlike several recent reports in rat, 5,6 our study did not find an internal pattern of organization for particular movements. Rather, the same movement was multiply represented within the map in a mosaic fashion similar to that previously reported in cat ${ }^{9}$ and monkey. ${ }^{9,34,35}$

One interesting finding was that stimulation delivered at threshold often elicited movements from two or more parts of the body. This overlap was particularly evident at the common border between the forelimb and hindlimb as well as at the border between the vibrissae and limbs. Our results are in agreement with those of Gioanni and Lamarche ${ }^{5}$ who reported an extensive overlap of the vibrissae with portions of the limbs and tail in rat, but unlike our findings, theirs do not support an overlap between the forelimb and hindlimb representations.

The possibility remains that the observed overlap results from current spread around the stimulating electrode. In many cases, currents less than or equal to $10 \mu \mathrm{A}$ were sufficient to elicit movements from more than one body part at the same stimulating site. It has been calculated that currents of $10 \mu \mathrm{A}$ are capable of spreading approximately 90 microns from the stimulating electrode $^{36}$ while lower currents produce less spread. Although cur-

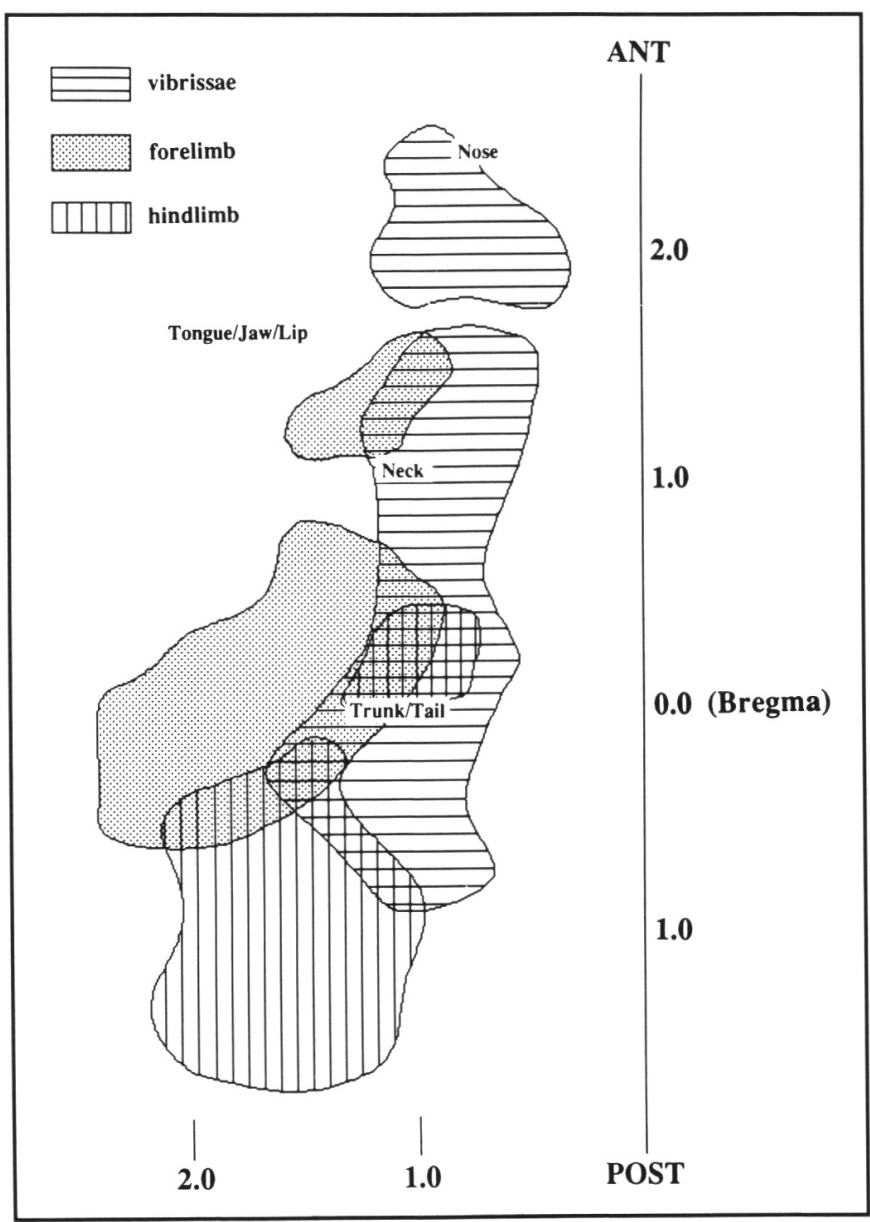

Figure 9-Idealized drawing of the location and distribution of the motor representation of individual body-parts as determined by ICMS. rent spread cannot be ruled out as an explanation for overlap, our results equally support the notion that in the fringe of a body part representation cortical neurons may coexist in close proximity to neighboring neurons serving a different body part.

The present results in mouse combined with previous results reported in rat suggest that these two species share a common plan of motor organization with regard to cytoarchitecture, corticospinal tract organization, and functional organization.

\section{ACKNOWLEDGEMENTS}

The authors are indebted to Dr. R.W. Dykes for commenting on the manuscript. We thank $M$. Waters for editing. This work was supported by NSF grant BNS-88-02766 to R.S.W. C.X.L. is a visiting scientist from China Medical University.

\section{REFERENCES}

1. Hall RD, Lindholm EP. Organization of motor and somatosensory neocortex in the albino rat. Brain Res 1974; 66: 23-38.

2. Sapienza S, Talbi B, Jacquemin J, et al. Relationship between input and output cells in motor and somatosensory cortices of the chronic awake rat. Exp Brain Res 1981; 43: 47-56.

3. Donoghue J, Wise SP. The motor cortex of the rat: cytoarchitecture and microstimulation mapping. J Comp Neurol 1982; 212: 76-88.

4. Sanderson K. Welker W, Shambes G. Reevaluation of motor cortex and sensorimotor overlap in cerebral cortex of albino rats. Brain Res 1984; 292: 251-260.

5. Gioanni Y, Lamarche M. A reappraisal of rat motor cortex organization by intracortical microstimulation. Brain Res 1985; 344: 49-61.

6. Neafsey EJ, Bold EL, Haas G, et al. The organization of the rat motor cortex: a microstimulation mapping study. Brain Res Rev 1986; 11: 77-96.

7. Asanuma $\mathrm{H}$, Sakata $\mathrm{H}$. Functional organization of a cortical efferent zone examined with focal depth stimulation in cat. J Neurophysiol 1967; 30: 35-54.

8. Stoney DS Jr, Thompson F, Asanuma H. Excitation of pyramidal tract cells by intracortical microstimulation: effective extent of stimulating current. J Comp Neurol 1967; 31: 659-669.

9. Asanuma $\mathrm{H}$, Rosen I. Topographical organization of cortical efferent system projecting to distal forelimb muscles in the monkey. Exp Brain Res 1972; 14: 243-256.

10. Woolsey TA, Van der Loos $\mathrm{H}$. The structural organization of layer IV in the somatosensory region (SI) of mouse cerebral cortex. Brain Res 1970; 17: 205-242.

11. Welker C, Woolsey TA. Structure of layer IV in the somatosensory neocortex of the rat: description and comparison with the mouse. J Comp Neurol 1974; 158: 437-453.

12. White EL, DeAmicis RA. Afferent and efferent projections of mouse Sml cortex which contains the posteromedial barrel subfield. J Comp Neurol 1977; 175: 455-482.

13. Porter LL, White EL. Afferent and efferent pathways of the vibrissal region of primary motor cortex in the mouse. J Comp Neurol 1983; 214: 279-289.

14. Simons DJ, Durham D, Woolsey TA. Functional organization of mouse and rat $\mathrm{SmI}$ barrel cortex following vibrissal damage on different postnatal days. Somatosens Res 1984; 1: 207-245.

15. Simons DJ, Woolsey TA. Functional organization in mouse barrel cortex. Brain Res 1979; 165: 327-332.

16. Brodmann K. Vergleichende Lokalisationslehre der Grosshirn-rinde in ihren Prinzipien dargestellt auf Grund des Zellenbause. Barth JA, Leipzig 1909.

17. Krieg WJS. Connections of the cerebral cortex. I. The albino rat. A Topography of the cortical areas. J Comp Neurol 1946; 84: 221 275.

18. Caviness VS Jr. Architectonic map of neocortex of the normal mouse. J Comp Neurol 1975; 164: 247-264.

19. Donoghue JP, Suner S, Sanes JN. Rapid reorganization of dynamic organization of the primary motor cortex output to target muscle groups. II. Rapid reorganizational shifts following motor nerve lesions. Exp Brain Res 1990 (in press). 
20. Sanes JN, Suner S, Donoghue JP. Dynamic organization of primary motor cortex output to target muscles in adult rats. I. Long-term patterns of reorganization following motor or mixed peripheral nerve lesions. Exp Brain Res 1990 (in press).

21. Chapin JK, Lin C-S. Mapping the body representation in the SI cortex of anesthetized and awake rats. J Comp Neurol 1984; 229: 199-213.

22. Terashima T, Inoue $K$, Inoue $Y$, et al. Distribution and morphol-ogy of corticospinal tract neurons in reeler mouse cortex by the retrograde HRP method. J Comp Neurol 1983; 218: 314-326.

23. Miller MW. The origin of corticospinal projection neurons in rat. Exp Brain Res 1987; 67: 339-351.

24. Leong $S$. Localizing the corticospinal neurons in neonatal, developing and mature albino rat. Brain Res 1983; 265: 1-9.

25. Hicks S, D'Amato C. Locating corticospinal neurons by retrograde axonal transport of horseradish peroxidase. Exp Neurol 1977; 56: $410-420$.

26. Wise $S$, Jones EG. Cells of origin and terminal distribution of descending projections of the rat somatic sensory cortex. J Comp Neurol 1977; 175: 129-158.

27. Nudo RJ, Masterton RB. Descending pathways to the spinal cord, III: Sites of origin of the corticospinal tract. J Comp Neurol 1990; 296: 559-583.

28. Andersen P, Hagan P, Phillips C, et al. Mapping by microstimulation of overlapping projections from area 4 to motor units of the baboon's hand. Proc R Soc Lond (Ser B) 1975; 188: 31-60.
29. Mcllwain J. Lateral spread of neural excitation during micro-stimulation in intermediate grey layer of cat's superior colliculus. $J$ Neurophysiol 1982; 47: 167-177.

30. Jankowska E, Padel Y, Tanaka R. The mode of activation of pyramidal tract cells by intracortical stimuli. J Physiol (Lond) 1975; 249: 617-636.

31. Jankowska E, Padel Y, Tanaka R. Projections of pyramidal tract cells to alpha-motorneurons innervating hindlimb muscles in the monkey. J Physiol (Lond) 1975; 249: 637-669.

32. Lemon $\mathrm{R}$. The output map of the primate motor cortex. Trends in Neurosci 1988; 11:501-506.

33. Neafsey E, Sievert C. A second forelimb motor area exists in rat frontal cortex. Brain Res 1982; 232: 151-156.

34. Samulack DD, Waters RS, Dykes RW, et al. Absence of responses to microstimulation at the hand-face border in baboon primary motor cortex. Can J Neurol Sci 1990; 17: 24-29.

35. Waters RS, Samulack DD, Dykes RW, et al. Topographic organization of baboon primary motor cortex: Face, hand, forelimb, and shoulder representation. Somatosens Mot Res 1990 (in press).

36. Stoney SD Jr, Thompson WD, Asanuma $\mathrm{H}$. Excitation of pyramidal tract cells by intracortical microstimulation: effective extent of stimulating current. J Neurophysiol 1968; 31: 659-669. 